2010s-18

\title{
La formation continue, un moyen de réduire les inégalités salariales entre hommes et femmes?
}

Nathalie Havet, Guy Lacroix

\begin{tabular}{c}
\hline Série Scientifique \\
Scientific Series
\end{tabular}

Montréal

Avril 2010

(C) 2010 Nathalie Havet, Guy Lacroix. Tous droits réservés. All rights reserved. Reproduction partielle permise avec citation du document source, incluant la notice (C).

Short sections may be quoted without explicit permission, if full credit, including () notice, is given to the source.
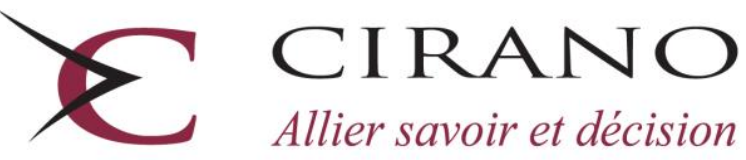

Allier savoir et décision

Centre interuniversitaire de recherche en analyse des organisations 


\section{CIRANO}

Le CIRANO est un organisme sans but lucratif constitué en vertu de la Loi des compagnies du Québec. Le financement de son infrastructure et de ses activités de recherche provient des cotisations de ses organisations-membres, d'une subvention d'infrastructure du Ministère du Développement économique et régional et de la Recherche, de même que des subventions et mandats obtenus par ses équipes de recherche.

CIRANO is a private non-profit organization incorporated under the Québec Companies Act. Its infrastructure and research activities are funded through fees paid by member organizations, an infrastructure grant from the Ministère du Développement économique et régional et de la Recherche, and grants and research mandates obtained by its research teams.

\section{Les partenaires du CIRANO}

Partenaire majeur

Ministère du Développement économique, de l'Innovation et de l'Exportation

\section{Partenaires corporatifs}

Banque de développement du Canada

Banque du Canada

Banque Laurentienne du Canada

Banque Nationale du Canada

Banque Royale du Canada

Banque Scotia

Bell Canada

BMO Groupe financier

Caisse de dépôt et placement du Québec

Fédération des caisses Desjardins du Québec

Gaz Métro

Hydro-Québec

Industrie Canada

Investissements PSP

Ministère des Finances du Québec

Power Corporation du Canada

Raymond Chabot Grant Thornton

Rio Tinto

State Street Global Advisors

Transat A.T.

Ville de Montréal

\section{Partenaires universitaires}

École Polytechnique de Montréal

HEC Montréal

McGill University

Université Concordia

Université de Montréal

Université de Sherbrooke

Université du Québec

Université du Québec à Montréal

Université Laval

Le CIRANO collabore avec de nombreux centres et chaires de recherche universitaires dont on peut consulter la liste sur son site web.

Les cahiers de la série scientifique (CS) visent à rendre accessibles des résultats de recherche effectuée au CIRANO afin de susciter échanges et commentaires. Ces cahiers sont écrits dans le style des publications scientifiques. Les idées et les opinions émises sont sous l'unique responsabilité des auteurs et ne représentent pas nécessairement les positions du CIRANO ou de ses partenaires.

This paper presents research carried out at CIRANO and aims at encouraging discussion and comment. The observations and viewpoints expressed are the sole responsibility of the authors. They do not necessarily represent positions of CIRANO or its partners. 


\title{
La formation continue, un moyen de réduire les inégalités salariales entre hommes et femmes?
}

\author{
Nathalie Havet ${ }^{*}$, Guy Lacroix ${ }^{\dagger}$
}

\author{
Résumé / Abstract
}

On continue en entreprise (formelle et informelle), en centrant l'analyse sur les différences entre sexes. Pour ce faire, les données françaises de l'enquête Formation continue 2000 sont mobilisées. On estime un modèle d'équations simultanées afin de tenir compte à la fois du phénomène de sélection endogène des pratiques de formation et des effets corrélés de l'hétérogéneité individuelle inobservable entre les différents types de formation et les salaires. Il ressort, qu'en France, le rendement d'une formation formelle est plus élevé pour les femmes que pour leurs homologues masculins et que le rendement d'une formation informelle est équivalent pour les deux sexes.

Mots clés : formations formelle et informelle, différences hommes/femmes, rendements des formations, équations simultanées.

Can Continuous Training Reduce the Gender Wage Gap?: This paper investigates the returns to formal and informal on-the-job training with an emphasis on gender wage differences. The analysis is based upon the French data set Formation continue 2000. We estimate a system of three simultaneous equations in order to take into account the endogenous selection in each type of training as well as the correlated effects of the unobserved heterogeneity between the training programs and the wages. Our results show that the return to formal training in France is higher for women and that the return to informal training is similar across gender.

Keywords: formal and informal training, gender differences, returns to training, simultaneous equations.

Codes JEL : J16, J24, J31

\footnotetext{
* GATE, 93, chemin des Mouilles B.P. 16769131 ECULLY. Tél : 04728660 83. Fax : 0472866090.

E-mail : havet@gate.cnrs.fr

$\dagger$ Département d'Economique, Pavillon J.A. De Sève, Québec, Québec, G1K 7P4, Canada.

Tél : 418-656-5122. Fax : 418-656-2707. E-mail : Guy.Lacroix@ecn.ulaval.ca
} 


\section{Introduction}

Dans la littérature macroéconomique, il y a un large consensus quant à l'influence des investissements en capital humain et de la formation professionnelle de la main d'œuvre sur le développement économique et sur la compétitivité d'un pays ${ }^{1}$. Selon cette logique, de nombreux pays de l'OCDE ont non seulement mis en place des politiques subventionnant les entreprises désireuses de parrainer de la formation au sein de leurs établissements ${ }^{2}$ mais aussi des lois imposant aux entreprises de financer de la formation continue. Par exemple, le système français de formation professionnelle a été développé, pour l'essentiel, sur le principe d'une obligation faite aux entreprises de former en permanence le plus grand nombre possible de travailleurs [Cahuc \& Zylberberg (2006)]. Mis en place dans sa forme actuelle au début des années soixantedix et largement réformé depuis, il établit le principe de "former ou payer" : les entreprises de plus de 10 salariés doivent, chaque année, affecter un minimum de 1,5\% (révisé à 1,6\% en 2004) de leur masse salariale à la formation continue et les entreprises dont les dépenses de formation sont néanmoins inférieures à ce seuil légal s'acquittent d'une taxe ou versent le complément à des organismes publics prestataires de formation.

Au niveau microéconomique, la formation continue est une stratégie essentielle en matière de ressources humaines pour les employeurs. Elle permet de pallier les déficits en compétence de base des individus, d'adapter les qualifications spécifiques des travailleurs à leurs besoins de court terme et de préparer les salariés aux profonds changements technologiques. Les entreprises cherchent par ce biais à améliorer l'efficacité productive de leur organisation. D'ailleurs, la plupart des études empiriques concluent que la formation continue est susceptible d'avoir des impacts non négligeables sur la performance de l'entreprise en termes de productivité [Bartel (1995), Black \& Lynch (1998), Barrett \& O'Connell (2001), Black \& Lynch (2001)] et de profits [Delame \& Kramarz (1997), OCDE (1995), Gauron (2000)]. Mais la formation professionnelle bénéficie-t-elle aussi aux salariés et en particulier a-t-elle une incidence positive sur leur niveau de rémunération?

D'un point de vue théorique, il n'y a pas de parfait consensus sur l'existence d'une relation systématique entre formation continue et augmentation de salaires due à une hausse de productivité. Certes, la théorie du capital humain initiée par Becker (1964) affirme que la formation continue en entreprise est un investissement générant un accroissement du niveau de qualifications des bénéficiaires, qui doit se traduire, à terme, par une hausse salariale. En effet, basée sur l'hypothèse de concurrence parfaite du marché du travail, elle admet que le salaire reflète

\footnotetext{
${ }^{1}$ Les nombreux modèles dans la lignée de ceux de la croissance endogène s'intéressent à cette question. Voir par exemple Coulombe, Tremblay \& Marchand (2004).

${ }^{2}$ C'est le cas par exemple dans la plupart des provinces canadiennes. L'Ontario octroie aux entreprises offrant de la formation continue des crédits d'impôts généreux. Le Québec, par l'entremise de sa Loi favorisant le développement et la reconnaissance des compétences de la main-d'œuvre, impose aux entreprises dont la masse salariale annuelle est de plus d'un million de dollars d'investir, au cours d'une même année civile, l'équivalent d'au moins $1 \%$ de cette masse salariale dans la réalisation d'activités de formation. À défaut d'investir cette somme, elles sont tenues de verser au Fonds de développement et de reconnaissance des compétences de la main-d'œuvre le montant résiduel.
} 
la productivité marginale du travailleur et que celle-ci est croissante avec le stock de connaissances accumulées. C'est pourquoi, elle prédit que l'investissement en formation professionnelle devrait se traduire automatiquement par une augmentation de salaire. Or dans la réalité, il existe des imperfections du mécanisme concurrentiel, remettant en cause cet argumentaire ${ }^{3}$. Par exemple, tout ou partie des investissements en formation réalisés par certaines entreprises découle de l'obligation légale et non d'une décision rationnelle de leur part. Il se peut donc que les formations offertes n'augmentent pas véritablement la productivité des salariés. Par ailleurs, si les employeurs sont peu nombreux sur un segment particulier du marché du travail, ils ont un pouvoir de monopsone qui leur offre la possibilité de fixer des salaires inférieurs à la productivité individuelle. Dans ce cas, même si la formation continue génère des gains de productivité, ces derniers ne seront pas intégralement répercutés sur les salaires [Acemoglu \& Pischke (1999)]. Les asymétries d'information en faveur des employeurs, c'est-à-dire l'avantage informationnel dont ils disposent sur les capacités de leurs propres salariés par rapport à leurs concurrents potentiels, peuvent aussi contribuer à affaiblir la relation entre productivité et salaire [Chang \& Wang (1996)]. C'est pourquoi, la rentabilité de la formation professionnelle est une question qui mérite d'être tranchée empiriquement.

Toutefois, l'évaluation statistique de l'impact de la formation professionnelle sur les salaires reste délicate. D'une part, les décisions concernant la formation professionnelle et les salaires se prennent souvent de manière concomitante. On peut penser que chaque travailleur décide de son investissement en formation continue en fonction de son rendement anticipé en termes de surcroît de salaire. Ce sont ainsi les salariés les plus efficaces qui ont intérêt à investir en formation et donc ceux qui seront potentiellement les mieux rémunérés. Par conséquent, une spécification économétrique correcte doit traiter adéquatement l'éventuelle endogénéité de la formation continue par rapport aux salaires : le cadre retenu dans de nombreuses études, qui consiste à estimer une équation de salaire mincérienne incluant une dichotomique qui vaut 1 si l'individu a suivi une formation et 0 sinon, conduit en général à des biais dans les rendements estimés et à des ordres de grandeur trop élevés ${ }^{4}$. D'autre part, il faut être capable de séparer le véritable impact de la formation continue de celui de caractéristiques inobservées, qui y seraient corrélées. En effet, la plupart des travaux sur la rentabilité salariale de la formation professionnelle disposaient d'informations précises, soit uniquement sur les caractéristiques et stratégies d'entreprises, soit uniquement sur les caractéristiques des salariés, mais rarement sur les deux dimensions en même temps. Or pour qu'une formation continue soit suivie, celle-ci doit être non seulement proposée par l'entreprise mais aussi acceptée par le travailleur. Il est donc fort probable que l'on associe à la formation continue une influence causale sur les salaires qui ne serait en fait que le reflet des caractéristiques inobservables, corrélées avec la formation continue, et donc omises dans la spécification économétrique.

\footnotetext{
${ }^{3}$ Pour plus de détails, voir Leuven (2005).

${ }^{4}$ Voir Bassanini, Booth, Brunello, Paola \& Leuven (2007) pour une revue de la littérature des estimations du rendement salarial de la formation continue pour les principaux pays de l'OCDE en fonction des méthodes économétriques utilisées.
} 
Les études empiriques récentes, soucieuses de tenir compte adéquatement du problème d'endogénéité, et/ou de l'hétérogénéité inobservée, trouvent en général que la formation continue a un effet faible, voir nul, sur le salaire [Voir Tableau 4.1 dans Bassanini et al. (2007, p.123)]. En particulier, Goux \& Maurin (2000) mettent en évidence, à partir de données françaises appariées employé-employeur de 1993, que les employés ayant suivi une formation professionnelle ne bénéficient pas d'un supplément de salaire significatif par rapport à leurs homologues n'ayant pas suivi de formation. Ils montrent que s'ils n'avaient pas tenu compte de la sélection endogène dans la participation aux stages de formation, le rendement estimé aurait été significatif et de l'ordre de 7\% au lieu d'être nul. En d'autres termes, leur étude confirme que ce sont les salariés les plus productifs qui suivent les stages de formation et qui obtiennent les plus fortes hausses de salaires, non pas du fait de leur participation à ces programmes, mais du fait de leurs caractéristiques personnelles. Fougère, Goux \& Maurin (2001) précisent cette analyse, à partir d'un échantillon d'hommes issus de la même base de données, en proposant une évaluation distincte du rendement de la formation selon que les salariés restent avec leur entreprise formatrice ou non. Pour les salariés ne changeant pas d'entreprise, ils retrouvent le résultat de Goux \& Maurin (2000) de rendement salarial nul de la formation. Pour ceux en changeant, ils concluent à un rendement significatif et positif de la formation, mais qui ne compenserait pas la perte de salaire occasionnée par le changement d'entreprise.

L'objectif de cet article est de compléter ces deux seuls travaux existant pour la France sur la rentabilité salariale de la formation professionnelle et qui sont basés sur des données qui commencent à dater. Néanmoins, cette question sera traitée en se focalisant sur une dimension supplémentaire : les différences de valorisation de la formation continue entre sexes. On cherchera à savoir si les hommes et les femmes ont un accès différencié à la formation en entreprise et si celle-ci joue le même rôle pour les deux sexes en termes d'augmentations salariales. En effet, il a été montré à plusieurs reprises que les femmes avaient moins de chances d'acquérir une formation professionnelle que leurs homologues masculins et que cette différence pouvait expliquer une proportion importante de l'écart salarial entre sexes [Corcoran \& Duncan (1979), Gronau (1988), Lynch (1992), Hill (2001)]. Néanmoins, les travaux de Lillard \& Tan (1992), Veum (1996), et très récemment de Turcotte, Léonard \& Montmarquette (2003) et Havet (2006) sur données canadiennes sont contradictoires puisqu'ils ne concluent à aucune différence entre sexes dans la probabilité de recevoir une formation en entreprise et dans sa rentabilité salariale. Il n'est donc pas superflu de quantifier le rendement de la formation sur des données françaises récentes pour avoir une vision actualisée des disparités perdurant entre hommes et femmes. L'utilisation de l'enquête Formation continue 2000, complémentaire à l'Enquête Emploi et réalisée par l'Insee, va nous permettre d'obtenir ce nouvel éclairage pour la France. Par ailleurs, cette enquête distingue la formation en situation de travail de la formation structurée, ce qui offre la possibilité d'examiner les liens entre formation continue formelle et informelle, de comparer leurs impacts respectifs sur les salaires et de savoir s'il existe des différences entre sexes dans la nature et les rendements des formations suivies. Jusque-là, la plupart des études se sont uniquement concentrées sur la formation structurée, faute de données disponibles sur les autres catégories de formations en entreprise. Pourtant, cette distinction semble primordiale puisque Havet (2006) montre qu'il existe au Canada des 
écarts de rendements entre ces différents types de formation, en termes d'accession à un poste à plus hautes responsabilités et en termes d'accroissement du niveau de rémunération : la formation structurée aurait notamment une rentabilité salariale plus élevée que la formation en situation d'emploi.

Le reste de cet article est organisé comme suit. La section 2 présente les données de l'enquête Formation continue 2000. La section 3 détaille le modèle économétrique retenu, qui est un système à 3 équations simultanées : la première équation décrit l'accès à la formation informelle, la seconde la participation à une formation formelle et la troisième correspond à une équation de salaire dans laquelle les deux mesures de formation continue sont introduites comme variables explicatives. Cette section précisera donc la spécification économétrique choisie ainsi que la stratégie d'estimation employée. Enfin les principaux résultats des estimations seront commentés dans la section 4.

\section{Les données}

\subsection{L'enquête Formation Continue 2000}

L'enquête Formation Continue 2000 est complémentaire à l'enquête Emploi annuelle de mars 2000, réalisée par l'Insee et le Céreq. Alors que la majorité des sources françaises sur la formation continue porte sur les entreprises, ces données analysent la formation continue en partant des individus, qu'ils aient effectué une formation ou non. Cette enquête est en effet adressée à un échantillon de répondants à l'Enquête Emploi, susceptibles de bénéficier de formation continue : âgés de 15 à 65 ans, ayant terminé leur formation initiale depuis au moins un an et n'effectuant pas leur service national au moment de l'enquête. Ces personnes ont été interrogées, sous forme d'entretiens en face-à-face, sur les formations suivies au cours de leur vie active, c'est-à-dire depuis la fin de leurs études, avec un questionnement rétrospectif plus détaillé sur les 14 mois précédant l'enquête (janvier 1999 - février 2000). Nous utiliserons ici uniquement ce dernier volet. A partir de l'Enquête Emploi 2000, on peut recueillir pour les personnes interrogées des données sur leurs salaires, leurs heures de travail, leur capital humain, les caractéristiques de leur emploi et autres informations démographiques habituellement recueillies dans les enquêtes auprès des ménages (âge, situation matrimoniale, niveau de scolarité, etc).

Une des originalités de cette enquête est de distinguer différents types de formation continue. Il est demandé aux enquêtés si au cours des quatorze derniers mois, ils ont suivi un des programmes de formation suivants : i) des stages, cours de formation continue, séminaires et conférences, qui désignent les formations se déroulant avec l'aide d'un formateur ou d'un intervenant spécialisé, dans un endroit distinct du poste de travail, ii) des formations en situation de travail, c'est-à-dire qui se déroulent sur le poste de travail ou un poste équivalent, avec l'appui d'un tuteur et en faisant appel aux outils de travail, iii) l'auto-formation qui regroupe des pratiques de formation où la personne se forme principalement seule, éventuellement avec des outils 
de formation particuliers ${ }^{5}$, iv) la formation en alternance, qui correspond à des contrats de travail spécifiques prévoyant une formation obligatoire dans un établissement scolaire. Toutes les actions de formation d'une durée au moins égale à trois heures sont retenues. Toutefois, les formations en alternance, répertoriées dans cette enquête, ne seront pas considérées ici comme une formation continue en tant que telle, puisque le contenu est fondamentalement différent des autres formes de formation et qu'elles relèvent autant d'une éducation initiale. En accord avec la littérature, nous désignerons dans la suite de l'étude par formation formelle le premier groupe de formation et nous regrouperons sous le nom de formation informelle les deux autres types de formation restants. Un aspect séduisant de l'enquête Formation Continue 2000 réside ainsi dans la possibilité d'examiner les liens entre formations formelle et informelle et de savoir s'il existe des différences entre sexes dans la nature des formations suivies.

Seuls les individus qui étaient actifs en 1999 et en 2000 et qui ne se déclaraient ni indépendants, ni employeurs, ni aide familial ou exploitant agricole ont été gardés. Notre échantillon final est alors constitué de 15059 salariés (8 027 hommes et 7032 femmes); ses principales caractéristiques sont décrites dans le Tableau 1.

\section{$2.2 \quad$ Formation formelle, formation informelle et salaires}

Selon les réponses apportées par les employés, un peu plus de la moitié d'entre eux $(52 \%)$ ont suivi une formation en entreprise du 1er janvier 1999 au 31 mars 2000. La proportion des employés ayant suivi une formation formelle (FCF) est de l'ordre de $42 \%$ contre $18 \%$ pour la formation informelle (FCI). On observe que $8 \%$ des employés ont acquis une combinaison des deux types de formation au cours des quatorze mois étudiés (Tableau 2).

Même si les taux d'accès aux différents types de formation, présentés dans le Tableau 2, semblent à première vue relativement proches entre sexes, les tests de contingence (Pearson, rapport de vraisemblance) concluent néanmoins à un écart significatif entre hommes et femmes en matière de formation. La proportion de femmes ayant suivi une formation en entreprise est très légèrement supérieure à celle des hommes (52,8\% contre 51,0\%). La différence entre sexes est principalement due à un pourcentage plus élevé de femmes ayant reçu une formation formelle (35\% contre $32 \%$ pour les hommes). Il est à noter que les femmes sont moins nombreuses que les hommes à avoir couplé ce type de formation à une formation informelle. En revanche, les formations suivies par les deux sexes semblent sensiblement de la même durée ${ }^{6}$ pour les hommes et pour les femmes et sont majoritairement de très courtes durées : environ $80 \%$ des formations professionnelles suivies auraient une durée inférieure à une semaine.

Le Tableau 3 met en évidence qu'il existe des différences de valorisation salariale entre les deux types de formation continue. Les individus ayant reçu une formation professionnelle ont des salaires horaires plus élevés que ceux n'en ayant pas reçu. Néanmoins, une formation informelle est associée à un salaire moyen supérieur à une formation formelle de l'ordre de

\footnotetext{
${ }^{5}$ L'auto-formation comprend les formations à distance.

${ }^{6}$ Les variables de durée de formation ne sont pas très bien renseignées.
} 


\begin{tabular}{|c|c|c|c|}
\hline & ENSEMBLE & HOMMES & FEMMES \\
\hline Participation aux programmes de formation (\%) & 51,88 & 51,05 & 52,83 \\
\hline Formation continue formelle & 41,68 & 40,92 & 42,55 \\
\hline Formation continue informelle & 18,37 & 18,96 & 17,69 \\
\hline \multicolumn{4}{|l|}{ Caractéristiques individuelles } \\
\hline Age moyen (2000) & 40,54 & 40,32 & 40,77 \\
\hline Nationalité étrangère (\%) & 2,96 & 3,72 & 2,08 \\
\hline Célibataire (\%) & 22,70 & 19,25 & 26,64 \\
\hline \multicolumn{4}{|l|}{ Ancienneté (\%) } \\
\hline$<1$ an & 5,41 & 6,2 & 4,52 \\
\hline $1-5$ ans & 24,69 & 24,82 & 24,54 \\
\hline $5-10$ ans & 18,59 & 18,04 & 19,21 \\
\hline$\geq 10$ ans & 51,31 & 50,94 & 51,73 \\
\hline \multicolumn{4}{|l|}{ Diplôme (\%) } \\
\hline Aucun diplôme & 19,02 & 21,30 & 16,41 \\
\hline Diplôme secondaire (BEPC) & 6,71 & 5,82 & 7,72 \\
\hline Certificat technique court (CAP-BEP) & 29,92 & 35,03 & 24,09 \\
\hline Baccalauréat & 15,08 & 12,94 & 17,52 \\
\hline $\mathrm{Bac}+2$ & 16,69 & 12,93 & 20,99 \\
\hline Supérieur à $\mathrm{Bac}+2$ & 12,58 & 11,97 & 13,27 \\
\hline \multicolumn{4}{|l|}{ Statut d'emploi (\%) } \\
\hline Contrat à durée indéterminée & 95,27 & 95,59 & 94,91 \\
\hline Temps partiel & 12,89 & 2,48 & 24,76 \\
\hline Bilan de compétences réalisé par un organisme spécialisé & 6,27 & 7,00 & 5,43 \\
\hline \multicolumn{4}{|l|}{ Profession } \\
\hline - Cadre & 15,56 & 19,16 & 11,46 \\
\hline - Profession intermédiaire & 28,48 & 26,99 & 30,12 \\
\hline - Employé & 28,68 & 11,74 & 48,03 \\
\hline - Ouvrier & 27,30 & 42,11 & 10,40 \\
\hline Salaire horaire moyen en 2000 (francs) & 67,81 & 73,05 & 61,83 \\
\hline \multicolumn{4}{|l|}{ Caractéristiques de l'entreprise (\%) } \\
\hline \multicolumn{4}{|l|}{ Mise en place de : } \\
\hline - une politique de réduction du temps de travail & 30,75 & 34,78 & 26,15 \\
\hline - nouveaux logiciels, matériels infomatiques ou autres équipements & 55,89 & 58,45 & 52,96 \\
\hline - une nouvelle organisation de travail & 40,29 & 44,69 & 35,28 \\
\hline \multicolumn{4}{|l|}{ Taille de l'entreprise } \\
\hline 0 à 9 travailleurs & 22,87 & 20,56 & 25,51 \\
\hline 10 à 99 travailleurs & 23,43 & 24,63 & 22,07 \\
\hline 100 à 499 travailleurs & 18,33 & 18,60 & 18,03 \\
\hline 500 travailleurs et plus & 35,36 & 36,22 & 34,39 \\
\hline Nombre d'observations & 15059 & 8027 & 7032 \\
\hline
\end{tabular}

TABleau 1: CARACtéRIstiques De L'ÉChantillon 


\begin{tabular}{lcccc}
\hline & $\begin{array}{c}\text { Uniquement } \\
\text { Formation formelle }\end{array}$ & $\begin{array}{c}\text { Uniquement } \\
\text { Formation informelle }\end{array}$ & $\begin{array}{c}\text { Les deux types } \\
\text { de formation }\end{array}$ & $\begin{array}{c}\text { Aucune } \\
\text { formation }\end{array}$ \\
\hline Ensemble & $33,51 \%$ & $10,20 \%$ & $8,17 \%$ & $48,12 \%$ \\
Hommes & $32,09 \%$ & $10,13 \%$ & $8,83 \%$ & $48,95 \%$ \\
Femmes & $35,14 \%$ & $10,28 \%$ & $7,41 \%$ & $47,17 \%$ \\
\hline \multicolumn{2}{l}{$\begin{array}{l}\text { Tests d'égalité des répartitions entre hommes et femmes } \\
\text { Pearson }\end{array}$} & $\chi^{2}=22,2366, \mathrm{Pr}=0,000$ & ddl=3, \\
Rapport de vraisemblance & $\chi^{2}=22,2694, \mathrm{Pr}=0,000$ & ddl=3, & \\
\hline
\end{tabular}

TABleau 2: TAuX D'ACCÈs À Une Formation CONTINUE Entre le 1ER JANVIER 1999 ET LE 31 MARS 2000, EN FONCTION DU SEXE

\begin{tabular}{lccccc}
\hline & $\begin{array}{c}\text { Uniquement } \\
\text { Formation formelle }\end{array}$ & $\begin{array}{c}\text { Uniquement } \\
\text { Formation informelle }\end{array}$ & $\begin{array}{c}\text { Les deux types } \\
\text { de formation }\end{array}$ & $\begin{array}{c}\text { Aucune } \\
\text { formation }\end{array}$ & $\begin{array}{c}\text { Ensemble } \\
\text { Ensemble }\end{array}$ \\
& 76,23 & 77,93 & 83,58 & 57,13 & 67,81 \\
Hommes & $(185,43)$ & $(265,70)$ & $(238,60)$ & $(105,14)$ & $(169,69)$ \\
& 79,85 & 85,78 & 92,80 & 62,39 & 73,04 \\
Femmes & $(122,66)$ & $(292,30)$ & $(311,39)$ & $(137,21)$ & $(177,09)$ \\
& 72,44 & 69,11 & 71,02 & 50,90 & 61,83 \\
& $(233,52)$ & $(232,06)$ & $(47,50)$ & $(42,18)$ & $(160,63)$ \\
\hline
\end{tabular}

Note : Écart-type entre parenthèses.

Tableau 3: Salaire horaire moyen en Mars 2000 (Francs)

$3,6 \%$. Cette situation globale cache des disparités de rentabilité des formations entre sexes. Les hommes ayant reçu une formation informelle ont un salaire moyen égal à 89,05 francs contre 82,65 francs pour ceux ayant suivi une formation formelle, soit un écart de 7,74\%. En revanche, parmi les femmes, la formation formelle semble avoir une meilleure valorisation salariale que la formation informelle, avec un supplément de rémunération de l'ordre de 3,3\%. Ainsi, les écarts salariaux entre sexes sont plus prononcés parmi les salariés ayant reçu une formation informelle alors qu'ils sont atténués parmi les salariés ayant suivi une formation formelle. Pour les individus ayant suivi une formation formelle, la différence de salaire hommes/femmes est de près de $15 \%$ du salaire féminin contre $18 \%$ sur l'ensemble de l'échantillon et $27 \%$ pour ceux ayant reçu une formation informelle.

Pour mieux apprécier les différences entre les groupes, la Figure 1 décrit les fonctions de densité des log-salaires lissées par un noyau d'Epanechnikov, pour les hommes et les femmes par type de formation. Elle fait ressortir que les fonctions de densité des femmes sont davantage concentrées vers la gauche que celles des hommes. Elle montre aussi que la fonction de densité des salaires des hommes ayant suivi une formation continue formelle a une queue de distribution beaucoup plus épaisse à droite que les autres groupes. Cela confirme qu'il y a une hétérogénéité importante parmi les sous-groupes ayant reçu une formation continue. Bien que suggestif, ce graphique ne fournit pas une preuve formelle que les différences entre les distributions salariales sont statistiquement significatives. C'est pourquoi, le Tableau 4 reporte une série de tests 


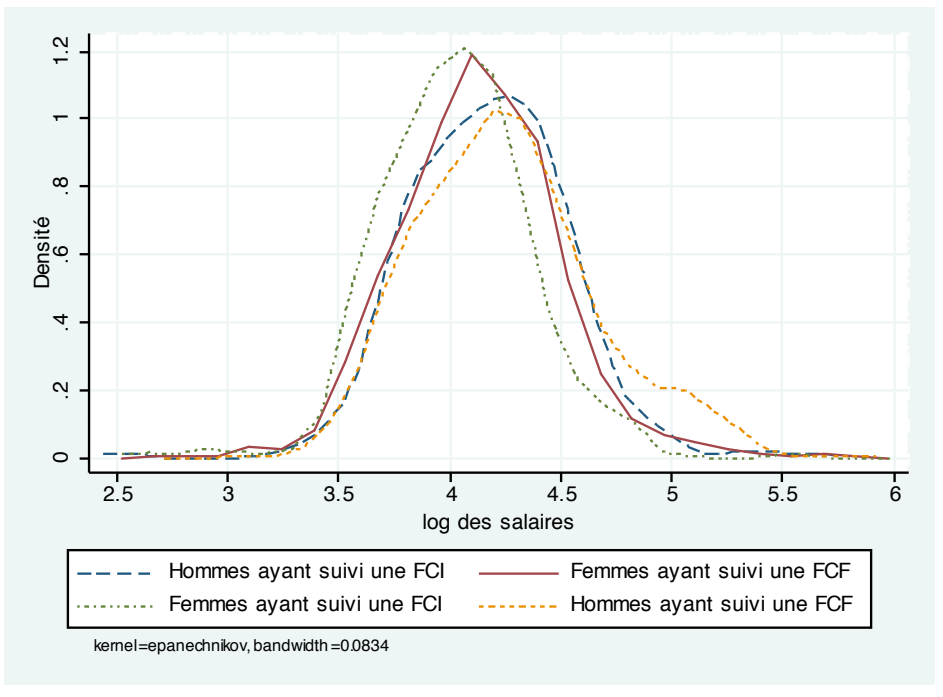

Figure 1: Distributions des salaires lissées par noyau d'Epanechnikov

d'égalité des fonctions de densité entre les sous-groupes pris deux à deux (tests de KolmogorovSmirnov). Selon ce tableau, toutes les distributions sont statistiquement distinctes.

\begin{tabular}{lccc}
\hline & Stat. $D$ & P. value & Nbre d'obs. \\
\hline Hommes avec FCF vs Femmes avec FCF & 0,1336 & 0,000 & 5047 \\
Hommes avec FCF vs Hommes avec FCI & 0,0817 & 0,001 & 3389 \\
Femmes avec FCF vs Femmes avec FCI & 0,1445 & 0,000 & 3194 \\
Hommes avec FCI vs Femmes avec FCI & 0,2210 & 0,000 & 1536 \\
\hline \multicolumn{4}{l}{ Note : Ce test compare H0 $: \hat{f}\left(x^{f}\right)=\hat{g}\left(x^{g}\right)$ contre H1 $: \hat{f}\left(x^{f}\right) \neq \hat{g}\left(x^{g}\right)$}
\end{tabular}

TAbleau 4: Test D'Égalité De Distributions : Statistiques De Kolmogorov-Smirnov

Ces premiers résultats montrent qu'il est indispensable de distinguer les différents types de formation professionnelle car leurs impacts en termes de carrières semblent être bien différents. Toutefois, ils ne sont pas suffisants pour conclure définitivement que la formation informelle exacerbe les disparités salariales entre sexes. Ils ne tiennent pas compte des effets conjoints des autres variables individuelles (poste, diplôme, etc.) sur l'accès aux formations et les salaires. D'où l'importance de recourir à une modélisation économétrique pour avoir une comparaison fiable des rendements des formations professionnelles, toutes choses étant égales par ailleurs. 


\section{Spécification économétrique et stratégie d'estimation}

Notre but est d'avoir une spécification économétrique qui modélise l'incidence de la formation professionnelle sur les salaires. Vu la nature des données de l'Enquête Formation Continue 2000, les retombées salariales évaluées correspondront à des rendements de court terme puisque les formations étudiées ont eu lieu au maximum quatorze mois avant la date d'observation du salaire. Toutefois, comme les formations professionnelles ont majoritairement des durées inférieures à une semaine, on s'attend à ce qu'elles soient rapidement valorisées et donc que leurs impacts se fassent ressentir au cours de ces quatorze mois. Par ailleurs, il est vraisemblable que l'impact de formations de très courtes durées ne soit que transitoire et par conséquent s'estompe au fil des années [Lengermann (1999)].

Le cadre retenu pour évaluer les rendements des différents types de formation ne peut se limiter à une équation de salaire mincérienne dans laquelle on inclut une variable dichotomique indiquant si l'individu a suivi ou non une formation formelle et une autre indiquant si l'individu a suivi ou non une formation informelle, en raison des problèmes d'endogénéité de ces variables et d'hétérogénéité inobservée. Ainsi, afin de tenir compte de la simultanéité des décisions de formations et de salaires, on propose d'estimer un système d'équations simultanées qui modélise conjointement les probabilités de suivre chaque type de formation (formelle et informelle) et leur influence sur les salaires. En outre, si de fortes capacités inobservées jouent à la fois sur les salaires et l'accès aux programmes de formation, on peut observer des salaires plus élevés pour les travailleurs qui ont reçu une formation même si celle-ci n'a pas d'impact réel sur les rémunérations. Par conséquent, afin de ne pas associer à la formation professionnelle une influence causale sur les salaires, qui ne serait que le reflet de caractéristiques inobservables, on suppose que les termes d'erreurs de notre système d'équations simultanées peuvent être corrélés entre eux : notre spécification permet ainsi d'obtenir des résultats robustes même si des caractéristiques individuelles omises influencent à la fois l'accès à la formation et les salaires.

\subsection{Le modèle économétrique}

Pour pallier ces problèmes économétriques, on propose un modèle à trois équations qui permet de tenir compte des biais de simultanéité : les deux premières équations décrivent l'accès aux deux types de formation continue et la dernière est une équation de salaire qui inclut FCF et FCI comme variables explicatives. Plus précisément, on note $y_{1 i}$ la variable dichotomique qui vaut 1 si le salarié $i$ a reçu une formation formelle entre janvier 1999 et mars 2000 et 0 sinon, et $y_{2 i}$ la variable dichotomique égale à 1 s'il a reçu une formation informelle sur la même période et 0 sinon, et enfin $w_{i}$ le salaire de l'employé $i$ en mars 2000. On considère le système suivant : 


$$
\begin{aligned}
y_{1 i} & =\left\{\begin{array}{lll}
1 & \text { si } & y_{1 i}^{*}=Z_{i} \gamma_{1}+u_{1 i}>0 \\
0 & \text { si } & y_{1 i}^{*}=Z_{i} \gamma_{1}+u_{1 i} \leqslant 0,
\end{array}\right. \\
y_{2 i} & =\left\{\begin{array}{lll}
1 & \text { si } & y_{2 i}^{*}=Z_{i} \gamma_{2}+u_{2 i}>0 \\
0 & \text { si } & y_{2 i}^{*}=Z_{i} \gamma_{2}+u_{2 i} \leqslant 0,
\end{array}\right. \\
\ln w_{i} & =X_{i} \beta+y_{1 i} \delta_{1}+y_{2 i} \delta_{2}+\alpha_{1}\left(y_{1 i} * \text { Sexe }_{i}\right)+\alpha_{2}\left(y_{2 i} * \text { Sexe }_{i}\right)+u_{3 i},
\end{aligned}
$$

où $\operatorname{Sexe}_{i}$ est une indicatrice qui vaut 1 si le salarié $i$ est un homme et 0 si c'est une femme. Les termes d'erreurs $\left(u_{1 i}, u_{2 i}, u_{3 i}\right)$ sont supposés être indépendants et identiquement distribués et suivre une loi normale trivariée de moyenne nulle et de matrice de variance-covariance $\Omega$ :

$$
\Omega=\left(\begin{array}{ccc}
1 & \sigma_{12} & \sigma_{13} \\
\sigma_{12} & 1 & \sigma_{23} \\
\sigma_{13} & \sigma_{23} & \sigma_{3}^{2}
\end{array}\right)
$$

Notre spécification modélise toutes les corrélations possibles entre les types de formation et les salaires, reflets d'éventuelles simultanéités dans les décisions. Par exemple, ce modèle offre l'avantage de prendre en compte le fait que les salariés peuvent combiner ou non la participation à une formation formelle et à une formation informelle, via l'estimation du coefficient de corrélation $\sigma_{12}$ entre les résidus de ces deux équations de participation. Puisque dans les modèles à variables discrètes des problèmes de cohérence logique [Maddala (1983)] rendent difficiles l'expression d'une simultanéité directe des décisions, on en tient compte ici par les coefficients de corrélation entre les caractéristiques non observables des salariés. Les décisions de suivre une formation formelle ou informelle peuvent être corrélées pour plusieurs raisons. La corrélation peut être positive si des liens de complémentarité existe entre les deux décisions, reflétant par exemple une volonté commune - mais inobservée - d'investir en capital humain. Mais la corrélation peut aussi être négative si les deux types de formation sont plutôt des substituts, constituant des outils différents permettant d'acquérir des connaissances similaires. Par ailleurs, on peut imaginer que certaines caractéristiques individuelles inobservables telles que l'habileté, la détermination ou l'envie de réussir du salarié influencent à la fois ses chances de participer à une formation continue et celles d'obtenir des salaires élevés. En conséquence, on permet des covariances non-nulles entre les équations de formation et de salaire $\left(\sigma_{13}\right.$ et $\left.\sigma_{23}\right)$.

Les équations (1) et (2) établissent que la participation aux deux types de formation continue dépend des mêmes caractéristiques, $Z$, mais leur influence peut différer entre formations formelle et informelle ( $\gamma_{1}$ est a priori différent de $\gamma_{2}$ ). Les paramètres $\delta_{1}$ et $\delta_{2}$ mesurent respectivement l'impact des formations formelle et informelle sur les salaires des femmes, tandis que les coefficients $\alpha_{1}$ et $\alpha_{2}$ captent l'écart de rendement salarial entre hommes et femmes de chacune des formations. D'après les statistiques descriptives, on peut s'attendre à ce que $\delta_{1}>\delta_{2}>0$, ou en d'autres termes que le rendement de la formation formelle soit supérieur à celui de la formation informelle chez les femmes. En revanche, la meilleure valorisation de la formation informelle chez les hommes sera vérifiée si $\left(\delta_{2}+\alpha_{2}\right)>\left(\delta_{1}+\alpha_{1}\right)$. 
L'identification des paramètres dans notre spécification ne nécessite aucune restriction d'exclusion car elle peut être réalisée par la forme fonctionnelle. Toutefois, notre base de données contient des variables qui expliquent la participation aux formations mais pas les salaires : tous les déterminants des salaires $X$ n'incluent pas tous les déterminants des formations $Z$ et $Z$ inclut des variables qui font référence à la situation de l'entreprise et du travailleur avant la formation, alors que $X$ inclut des variables décrivant la situation en 2000. C'est en soi suffisant pour identifier les paramètres $\delta$ et $\alpha$. Plus précisément, on retient comme variables explicatives $Z$ de la participation à une formation, non seulement des caractéristiques relatives au salarié (sexe, nationalité, nombre d'enfants, ancienneté, expérience, diplôme, type et statut de l'emploi occupé) mais aussi certaines caractéristiques relatives à l'entreprise (taille, branche d'activité). Des variables reflétant les stratégies d'organisation et de gestion de la main d'œuvre sont aussi introduites. Notamment, l'enquête Formation Continue 2000 nous indique si au cours des quatorze derniers mois, il y a eu ou non dans le service ou l'équipe du salarié : i) un aménagement ou une réduction du temps de travail ; ii) la mise en place de nouveaux logiciels, matériels informatiques, autres équipements ou techniques de production; iii) la mise en place d'une nouvelle organisation de travail (partage des tâches différent, application de normes qualité ou sécurité). Il ne serait pas étonnant que les travailleurs appartenant à un service qui a mis en place de nouveaux équipements ou un nouveau système de répartition des tâches, soient plus susceptibles de suivre une formation, en particulier pour s'adapter à leur nouvel environnement de travail. Enfin, on a retenu comme déterminant des deux formations une variable dichotomique qui vaut 1 si le salarié a bénéficié d'un bilan de compétences réalisé par un organisme spécialisé dans les quatorze derniers mois et 0 sinon. On s'attend a priori à ce qu'elle joue positivement sur la probabilité de suivre une formation puisque le bilan de compétences a pu pointer certaines connaissances à approfondir afin d'avoir une meilleure évolution de carrière. L'équation de salaire intègre les déterminants traditionnels tels que des variables reflétant le capital humain du salarié (ancienneté, expérience, diplôme), sa situation familiale (en couple, nombre d'enfants), le type d'emploi occupé (temps partiel, CDI, profession) et les caractéristiques de son établissement (branche d'activité, taille de l'entreprise).

\subsection{La technique d'estimation}

Il existe plusieurs méthodes d'estimation possibles pour le modèle complet défini par les équations (1), (2), (3) et (4). Il est possible d'adopter une stratégie en deux étapes, c'est-àdire d'estimer premièrement les paramètres des règles de participations aux programmes de formation en utilisant un modèle probit bivarié et deuxièmement d'estimer l'équation de salaire en utilisant les erreurs prédites comme variables explicatives supplémentaires afin de contrôler la sélection endogène des participants aux formations [Goux \& Maurin (2000)]. Cependant, on préfère ici estimer tous les paramètres simultanément en utilisant la technique du maximum de vraisemblance à information complète, qui donne des estimateurs plus efficaces. 
La fonction de vraisemblance associée à notre modèle peut s'écrire comme suit :

$$
\begin{aligned}
L\left(y_{1}, y_{2}, w \mid X, Z\right)=\prod_{i=1}^{n} & \left\{\left[\int_{-\infty}^{-Z_{i} \gamma_{1}} \int_{-\infty}^{-Z_{i} \gamma_{2}} \phi_{3}\left(u_{1 i}, u_{2 i}, \frac{u_{3 i}}{\sigma_{3}} ; \Omega\right) d u_{1 i} d u_{2 i}\right]^{\left(1-y_{1 i}\right) \cdot\left(1-y_{2 i}\right)}\right. \\
\times & {\left[\int_{-Z_{i} \gamma_{1}}^{+\infty} \int_{-\infty}^{-Z_{i} \gamma_{2}} \phi_{3}\left(u_{1 i}, u_{2 i}, \frac{u_{3 i}}{\sigma_{3}} ; \Omega\right) d u_{1 i} d u_{2 i}\right]^{y_{1 i} \cdot\left(1-y_{2 i}\right)} } \\
\times & {\left[\int_{-\infty}^{-Z_{i} \gamma_{1}} \int_{-Z_{i} \gamma_{2}}^{+\infty} \phi_{3}\left(u_{1 i}, u_{2 i}, \frac{u_{3 i}}{\sigma_{3}} ; \Omega\right) d u_{1 i} d u_{2 i}\right]^{\left(1-y_{1 i}\right) \cdot y_{2 i}} } \\
\times & {\left.\left[\int_{-Z_{i} \gamma_{1}}^{+\infty} \int_{-Z_{i} \gamma_{2}}^{+\infty} \phi_{3}\left(u_{1 i}, u_{2 i}, \frac{u_{3 i}}{\sigma_{3}} ; \Omega\right) d u_{1 i} d u_{2 i}\right]^{y_{1 i} \cdot y_{2 i}}\right\} }
\end{aligned}
$$

où $\phi_{3}$ est la fonction de densité d'une loi normale trivariée standardisée et $u_{3 i}=\ln w_{i}-X_{i} \beta-$ $y_{1 i} \delta_{1}-y_{2 i} \delta_{2}-\alpha_{1}\left(y_{1 i} * \operatorname{Sexe}_{i}\right)-\alpha_{2}\left(y_{2 i} *\right.$ Sexe $\left._{i}\right)$.

Par définition, la matrice de variance-covariance $\Omega$ doit être symétrique et définie positive. Afin de garantir ces propriétés pour la matrice estimée, la décomposition de Cholesky est appliquée lors de l'estimation. En d'autres termes, on écrit $\Omega=C C^{\prime}$ où $C$ est une matrice triangulaire inférieure :

$$
C=\left(\begin{array}{ccc}
c_{1} & 0 & 0 \\
c_{2} & c_{3} & 0 \\
c_{4} & c_{5} & c_{6}
\end{array}\right)
$$

Les contraintes de normalisation à l'unité des variances $\sigma_{1}^{2}$ et $\sigma_{2}^{2}$ impliquent les contraintes suivantes sur la matrice de Cholesky $C: c_{1}=1$ et $c_{2}=\sqrt{1-c_{3}^{2}}$. Ainsi, dans la pratique, ce sont les éléments $c_{j},(j=3, \ldots, 6)$ de la matrice de Cholesky qui seront directement estimés. Les éléments de la matrice $\Omega$ sont ensuite calculés en effectuant le produit $\hat{\Omega}=\hat{C} \hat{C}^{\prime}$.

\section{Résultats}

Les paramètres estimés de la matrice de variance-covariance $\Omega$ sont reportés dans le Tableau 5 et ceux des équations de formations et de salaires sont présentés dans le Tableau 6. On commencera par mettre en évidence l'importance des corrélations existantes entre les termes d'erreurs des différentes équations de notre modèle. Le reste de nos commentaires se concentrera sur les trois questions qui nous intéressent particulièrement, à savoir : 1) les liens entre les deux types de formation continue ; 2) les différences de rendement existant entre formations continues formelle et informelle; 3 ) la valorisation différenciée entre hommes et femmes des formations. 


\subsection{Hétérogénéité inobservée}

Le Tableau 5 révèle l'importance des corrélations existantes entre les termes d'erreurs des différentes équations. Les estimations indiquent que les équations de formation sont significativement et fortement corrélées $\left(\hat{\sigma}_{12}=-0,106\right)$. Certaines caractéristiques individuelles inobservables jouent ainsi simultanément sur les chances du salarié de suivre une formation formelle et sur celles de suivre une formation informelle, mais en sens opposé. Cela laisse penser que la probabilité de suivre une formation formelle influence négativement la probabilité de suivre une formation informelle durant la même période et inversement. Par conséquent, des liens de substitution existeraient entre les deux types de formation continue. Il était en tout cas indispensable d'estimer simultanément les équations de participation aux formations, comme l'avait déjà mis en évidence Havet (2006). Toutefois, sur données canadiennes, Havet (2006) obtenait que les deux types de formation étaient davantage complémentaires que substituables puisque la corrélation alors estimée était significative et positive. Cette différence entre la France et le Canada pourrait tenir de la spécificité du système français de formation professionnelle : afin de respecter l'obligation légale de formation, un certain nombre d'entreprises étalent probablement les formations données aux salariés dans le temps; il est plus pertinent pour elles d'en offrir une par année que plusieurs la même année.

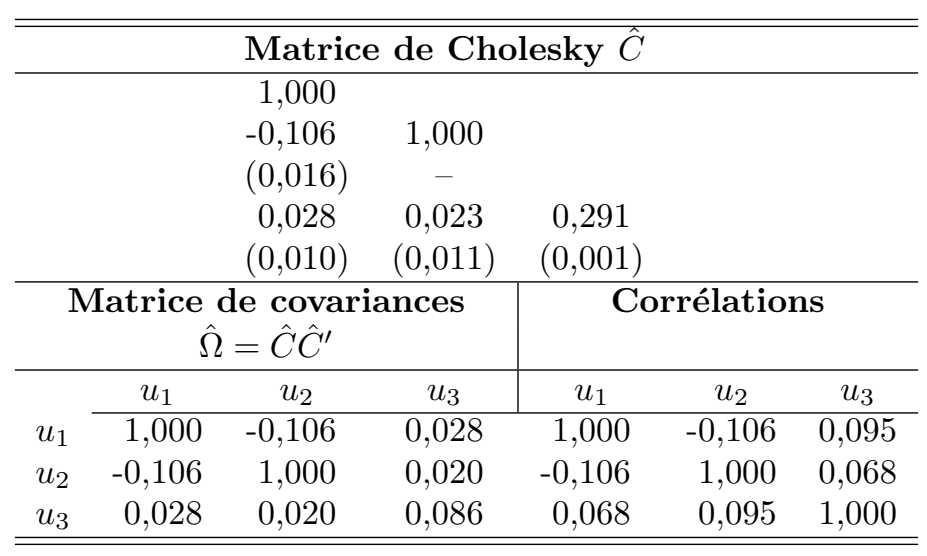

Tableau 5: Matrices de Cholesky et de covariances

Les covariances estimées $\hat{\sigma}_{13}$ et $\hat{\sigma}_{23}$ sont positives et significativement différentes de zéro. Des déterminants inobservés de la participation aux programmes de formation continue (formelle et informelle) semblent aussi être des déterminants des salaires individuels. Les salariés à fortes rémunérations ont plus de chances de participer à un programme de formation que les autres salariés. Goux \& Maurin (2000) ont mis en évidence des résultats similaires avec leur enquête française de 1993. La significativité de ces covariances suggère en outre que la seule correction de l'endogénéité des variables de formation par la méthode usuelle en deux étapes n'est pas suffisante pour avoir une bonne estimation des rendements salariaux de la formation 
professionnelle. Puisque les deux covariances $\hat{\sigma}_{13}$ et $\hat{\sigma}_{23}$ sont de même ampleur, cette sélection semble similaire quelle que soit la nature de la formation.

\subsection{Des différences d'accès et de rendements entre formations continues}

\subsubsection{Les déterminants des formations continues formelle et informelle}

Commençons par regarder les résultats des équations de formation. Les deux probabilités de recevoir une formation dépendent fortement du niveau d'éducation et de la position du salarié au sein de la hiérarchie des emplois. Plus les salariés ont un diplôme élevé, plus ils ont de chances d'obtenir une formation continue. La formation professionnelle est donc complémentaire à celle dispensée par le système scolaire. Elle permet peu aux salariés les moins diplômés de rattraper leur retard. De façon similaire, les efforts de formation ne sont pas concentrés sur les emplois au bas de l'échelle hiérarchique, mais sur ceux du milieu et du haut de la pyramide. Les ouvriers ont, toutes choses étant égales par ailleurs, des taux d'accès aux formations continues plus faibles. Ces résultats ne viennent que renforcer ceux de nombreuses études empiriques menées dans diverses pays qui s'accordaient à considérer que l'accès et les rendements de la formation sont significativement plus faibles pour les personnes les moins qualifiées [Bassanini et al. (2007), Heckman (2000)]. Un autre résultat classique est d'obtenir que les formations sont, de façon significative, plus fréquemment offertes aux salariés des grandes entreprises.

Contrairement à certains résultats antérieurs de la littérature, l'ancienneté joue peu sur la participation aux deux types de formation continue : elle n'influence pas significativement l'accès à la formation formelle alors que seuls les salariés avec plus de 10 ans d'ancienneté dans la même entreprise ont une probabilité plus élevée de recevoir une formation informelle que les autres. Cela suggère que la formation formelle serait un réel processus continu qui se développe, de manière ininterrompue, tout au long de la vie active et non un processus focalisé sur les jeunes entrants dans l'entreprise. Le fait que la formation informelle soit plus intensive parmi les salariés avec au moins 10 ans d'ancienneté pourrait révéler que ce type de formation est plus axé sur la mise à jour de toutes sortes de capacités et de connaissances spécifiques au poste de travail, comme par exemple l'apprentissage de nouveaux logiciels auxquels les plus jeunes ont pu s'initier lors de leur formation initiale. Toutefois, à ancienneté équivalente, les salariés les plus âgés ont une probabilité légèrement plus faible de suivre une formation puisque les coefficients associés à l'expérience sont statistiquement significatifs et négatifs.

Comme attendu, les salariés ayant bénéficié d'un bilan de compétences par un organisme spécialisé sont plus susceptibles de participer aux deux types de formation continue. De plus, les formations formelles et informelles sont plus fréquentes parmi les salariés des entreprises ayant mis en place une nouvelle organisation du travail et de nouveaux équipements dans les quatorze derniers mois. En revanche, les entreprises ayant adopté une réduction du temps de travail offrent, toutes choses étant égales par ailleurs, moins de formation formelle mais plus de formation informelle. On peut penser qu'une politique d'aménagement et de réduction du 


\begin{tabular}{|c|c|c|c|c|c|c|}
\hline \multirow[b]{2}{*}{ Variables Explicatives } & \multicolumn{2}{|c|}{$\begin{array}{c}\text { FORMATION } \\
\text { FORMELLE }\end{array}$} & \multicolumn{2}{|c|}{$\begin{array}{l}\text { FORMATION } \\
\text { INFORMELLE }\end{array}$} & \multicolumn{2}{|c|}{ SALAIRES } \\
\hline & Param & Ecart-type & Param & Ecart-type & Param & Ecart-type \\
\hline Constante & $-2,332$ & $(0,413)$ & $-2,122$ & $(0,455)$ & 3,405 & $(0,026)$ \\
\hline Sexe $($ homme $=1)$ & 0,019 & $(0,031)$ & 0,018 & $(0,034)$ & 0,137 & $(0,008)$ \\
\hline Nationalité étrangère & 0,207 & $(0,079)$ & 0,026 & $(0,091)$ & 0,018 & $(0,015)$ \\
\hline Couple & & & 0,019 & $(0,007)$ & & \\
\hline \multicolumn{7}{|l|}{ Nombre d'enfants } \\
\hline$<3$ ans & $-0,177$ & $(0,038)$ & $-0,039$ & $(0,042)$ & 0,020 & $(0,010)$ \\
\hline$[3-6[$ ans & 0,044 & $(0,030)$ & 0,048 & $(0,034)$ & 0,030 & $(0,007)$ \\
\hline$[6-18[$ ans & 0,009 & $(0,013)$ & $-0,021$ & $(0,015)$ & 0,016 & $(0,003)$ \\
\hline Expérience potentielle & $-0,012$ & $(0,002)$ & $-0,012$ & $(0,002)$ & 0,006 & $(0,000)$ \\
\hline \multicolumn{7}{|l|}{ Ancienneté (Réf $=<1$ an) } \\
\hline $1-5$ ans & $-0,042$ & $(0,039)$ & 0,048 & $(0,044)$ & $-0,033$ & $(0,012)$ \\
\hline 5-10 ans & 0,007 & $(0,040)$ & 0,073 & $(0,045)$ & 0,008 & $(0,013)$ \\
\hline$\geq 10$ ans & 0,021 & $(0,038)$ & 0,185 & $(0,042)$ & 0,100 & $(0,012)$ \\
\hline \multicolumn{7}{|l|}{ Diplôme (Réf = aucun diplôme) } \\
\hline Diplôme secondaire (BEPC) & 0,202 & $(0,055)$ & 0,048 & $(0,065)$ & 0,113 & $(0,011)$ \\
\hline Certificat technique court (CAP-BEP) & 0,218 & $(0,039)$ & 0,112 & $(0,046)$ & 0,106 & $(0,008)$ \\
\hline Baccalauréat & 0,385 & $(0,051)$ & 0,217 & $(0,058)$ & 0,184 & $(0,010)$ \\
\hline $\mathrm{Bac}+2$ & 0,465 & $(0,059)$ & 0,269 & $(0,067)$ & 0,257 & $(0,011)$ \\
\hline Supérieur à $\mathrm{Bac}+2$ & 0,479 & $(0,074)$ & 0,173 & $(0,084)$ & 0,347 & $(0,012)$ \\
\hline \multicolumn{7}{|c|}{ Profession (Réf $=$ Profession intermédiaire) } \\
\hline Cadre & 0,031 & $(0,051)$ & $-0,072$ & $(0,055)$ & 0,302 & $(0,009)$ \\
\hline Employé & $-0,256$ & $(0,037)$ & 0,047 & $(0,042)$ & $-0,169$ & $(0,008)$ \\
\hline Ouvrier & $-0,428$ & $(0,046)$ & $-0,169$ & $(0,051)$ & $-0,226$ & $(0,009)$ \\
\hline \multicolumn{7}{|l|}{ Statut de l'emploi } \\
\hline CDI & 0,125 & $(0,052)$ & $-0,124$ & $(0,059)$ & 0,130 & $(0,014)$ \\
\hline Temps partiel & $-0,054$ & $(0,037)$ & $-0,090$ & $(0,042)$ & $-0,028$ & $(0,008)$ \\
\hline Réalisation d'un bilan de compétences & 0,371 & $(0,045)$ & 0,341 & $(0,046)$ & & \\
\hline Formation continue formelle (FCF) & & & 0,072 & $(0,018)$ & & \\
\hline Formation continue formelle $*$ Sexe & & & $-0,039$ & $(0,011)$ & & \\
\hline Formation continue informelle (FCI) & & & 0,050 & $(0,021)$ & & \\
\hline Formation continue informelle $*$ Sexe & & & $-0,014$ & $(0,013)$ & & \\
\hline
\end{tabular}

TABleau 6: Les DÉterminants Des FORMations CONTINUES FORMELle ET INFORMELLE ET DES SALAIRES

temps de travail exige des salariés d'être plus polyvalents et ainsi d'apprendre par exemple une partie des tâches de leurs collègues pour être opérationnels même en leur absence. Or, cet apprentissage très spécifique aux outils de travail est probablement dispensé par un collègue ou un supérieur et se fait donc davantage par de la formation en situation de travail que de 


\begin{tabular}{|c|c|c|c|c|c|c|}
\hline & \multicolumn{2}{|c|}{$\begin{array}{l}\text { FORMATION } \\
\text { FORMELLE }\end{array}$} & \multicolumn{2}{|c|}{$\begin{array}{l}\text { FORMATION } \\
\text { INFORMELLE }\end{array}$} & \multicolumn{2}{|c|}{ SALAIRES } \\
\hline Variables Explicatives & Param & Ecart-type & Param & Ecart-type & Param & Ecart-type \\
\hline Réduction du temps de travail & $-0,081$ & $(0,027)$ & 0,102 & $(0,029)$ & & \\
\hline Nouveaux équipements & 0,237 & $(0,025)$ & 0,351 & $(0,029)$ & & \\
\hline Nouvelle organisation de travail & 0,206 & $(0,025)$ & 0,137 & $(0,028)$ & & \\
\hline \multicolumn{7}{|l|}{ Secteur d'activité (Réf = lndustrie) } \\
\hline Agriculture et industrie agroalimentaire & 0,017 & $(0,049)$ & $-0,030$ & $(0,052)$ & $-0,008$ & $(0,011)$ \\
\hline Construction & 0,082 & $(0,065)$ & $-0,255$ & $(0,080)$ & $-0,027$ & $(0,015)$ \\
\hline Commerce & 0,133 & $(0,046)$ & $-0,176$ & $(0,052)$ & $-0,056$ & $(0,011)$ \\
\hline Transport & 0,232 & $(0,059)$ & $-0,089$ & $(0,070)$ & 0,078 & $(0,012)$ \\
\hline Immobilier et services financiers & 0,310 & $(0,058)$ & $-0,132$ & $(0,063)$ & $-0,006$ & $(0,014)$ \\
\hline Services aux entreprises & 0,168 & $(0,047)$ & $-0,186$ & $(0,051)$ & $-0,053$ & $(0,012)$ \\
\hline Services aux particuliers & 0,076 & $(0,070)$ & $-0,237$ & $(0,086)$ & $-0,053$ & $(0,012)$ \\
\hline Education et santé & 0,219 & $(0,049)$ & $-0,149$ & $(0,054)$ & $-0,058$ & $(0,012)$ \\
\hline Administration & 0,288 & $(0,053)$ & 0,012 & $(0,058)$ & $-0,055$ & $(0,012)$ \\
\hline Secteur public & 0,128 & $(0,034)$ & $-0,039$ & $(0,039)$ & 0,034 & $(0,008)$ \\
\hline \multicolumn{7}{|c|}{ Taille de l'entreprise (Réf $=0$ à 9 salariés) } \\
\hline 10 à 99 salariés & 0,0215 & $(0,036)$ & 0,018 & $(0,043)$ & 0,062 & $(0,008)$ \\
\hline 100 à 499 salariés & 0,133 & $(0,039)$ & 0,169 & $(0,045)$ & 0,092 & $(0,009)$ \\
\hline 500 salariés et plus & 0,296 & $(0,037)$ & 0,173 & $(0,042)$ & 0,143 & $(0,008)$ \\
\hline \multicolumn{7}{|c|}{ Taille de la zone urbaine (Réf $=$ Zone rurale) } \\
\hline$<20000$ habitants & $-0,039$ & $(0,034)$ & 0,010 & $(0,039)$ & 0,014 & $(0,008)$ \\
\hline [20 $000-200000[$ habitants & 0,129 & $(0,032)$ & $-0,025$ & $(0,036)$ & 0,024 & $(0,008)$ \\
\hline 200000 habitants et plus & $-0,030$ & $(0,033)$ & $-0,001$ & $(0,037)$ & 0,034 & $(0,008)$ \\
\hline Agglomération parisienne & $-0,050$ & $(0,044)$ & $-0,271$ & $(0,052)$ & 0,137 & $(0,009)$ \\
\hline Log-vraisemblance & \multicolumn{6}{|c|}{$-18231,18$} \\
\hline Nombre d'observations & \multicolumn{6}{|c|}{15059} \\
\hline
\end{tabular}

TABleau 6:

Les déterminants des formations continues formelle et informelle et des salaires (Suite)

la formation formelle. En tout cas, les formations semblent faire partie d'une politique globale de l'entreprise, qui veut voir ses salariés acquérir des compétences plus élargies en matière d'organisation collective du travail, de relation avec la clientèle ou de gestion du temps de travail.

La différence la plus marquée entre les deux types de formation continue est que le fait d'avoir un contrat à durée limitée diminue ses chances de recevoir une formation formelle mais accroît celles d'avoir suivi une formation informelle. Il y aurait donc des inégalités d'accès à la formation formelle alors que selon la législation française, les salariés, quel que soit le statut de leur emploi, devraient bénéficier formellement des mêmes dispositions. Les travailleurs précaires, les plus exposés à une mobilité forcée, se verraient davantage proposer des formations informelles au détriment de formations formelles. Or, on peut craindre que les formations informelles, en grande partie dispensées en situation de travail et donc relativement spécifiques à l'entreprise, aient un caractère moins qualifiant et ouvrent moins de perspectives. En effet, les formations informelles visent essentiellement l'adaptation à l'emploi tandis que les formations formelles sont davantage associées à l'obtention d'un diplôme ou d'une qualification reconnue et 
donc à des connaissances plus transférables dans d'autres entreprises. Pourtant, l'acquisition de nouvelles qualifications est un atout essentiel pour le déroulement de la carrière des travailleurs précaires, car elle est le gage de leur adaptabilité aux exigences des entreprises susceptibles de les embaucher une fois leur contrat arrivé à terme. Perez \& Thomas (2005), qui ont étudié plus finement l'incidence de la précarité sur les opportunités d'accès à la formation en France, concluaient que connaître une trajectoire d'emploi précaire pénalise la participation à la formation continue au regard des parcours d'emploi stable. Néanmoins, nos résultats montrent que leurs conclusions méritent d'être nuancées en fonction du type de formation suivie. Pour le Royaume-Uni, Arulampalam \& Booth (1998) faisaient des constatations similaires. Le fait de travailler à temps partiel ne serait pas un handicap pour avoir accès à la formation formelle mais en revanche diminuerait les chances d'une formation informelle.

Certains secteurs d'activités sont plus propices au suivi d'une formation : les salariés ont plus de chances de suivre une formation formelle dans l'administration, le secteur bancaire, les assurances et autres activités financières et immobilières et le secteur des transports, et une formation informelle là-encore dans l'administration et les transports mais aussi dans le secteur industriel et agro-alimentaire. En revanche, l'appartenance au secteur des services aux particuliers s'accompagne d'une moindre probabilité d'accès aux deux types de formation.

\subsubsection{Incidence positive des formations continues sur les salaires}

Les deux dernières colonnes du Tableau 6 montrent que de nombreuses variables sont statistiquement significatives et ont l'effet attendu sur les salaires. Par exemple, les salaires sont croissants avec le niveau de scolarité, l'expérience professionnelle, la taille de l'établissement ou encore la taille de l'unité urbaine de résidence. Mais concentrons nous plus spécifiquement sur les rendements salariaux des deux formes de formations continues.

Nos résultats indiquent que la participation à une formation formelle et celle à une formation informelle ont des retombées significatives et positives sur le niveau des rémunérations. Contrairement à Leuven \& Oosterbeek (2008), Bassanini et al. (2007), Pischke (2001) et Goux \& Maurin (2000), même après avoir pris en compte les déterminants inobservables de la participation aux formations corrélés avec les salaires, on trouve que les salariés ayant suivi une formation continue bénéficieraient d'un supplément de salaire horaire par rapport à ceux n'en ayant pas suivi. Le gain salarial associé à la formation continue serait compris entre 3,5\% et $7 \%$.

Même si les rendements de la participation à une formation continue obtenus dans la littérature sont difficilement comparables entre eux du fait de différences dans les définitions retenues pour les formations et les périodes considérées, on peut néanmoins noter que nos résultats sont cohérents avec plusieurs études récentes mettant en évidence une incidence positive et significative des formations continues sur les salaires [Blundell \& Meghir (1999), Booth \& Bryan (2007), Regner (2002), Kuckulenz \& Zwick (2003), Schone (2004), Gerfin (2004), Bassanini et al. (2007)]. 
Nos évaluations correspondent plutôt à des valeurs moyennes dans la littérature empirique ${ }^{7}$. Elles sont du même ordre de grandeur que celles de plusieurs études réalisées sur données anglaises. Par exemple, Blundell, Dearden \& Meghir (1996) ont obtenu, à partir d'estimations à effets fixes et par l'approche des variables instrumentales, des rendements de la formation de l'ordre de $7 \%$ à $9 \%$ pour les hommes au Royaume-Uni. Plus récemment, Booth \& Bryan (2007) ont mis en évidence que le fait d'avoir reçu une formation financée par l'entreprise était associé à un supplément salarial variant de $2,4 \%$ à $7,5 \%$ selon que le salarié restait ou non avec le même employeur. Toutefois, l'influence des formations continues semble distincte entre hommes et femmes.

\subsection{Des inégalités entre hommes et femmes face aux formations continues?}

Il nous reste effectivement à répondre à notre question principale, c'est-à-dire à savoir si les hommes et les femmes ont un accès différencié à la formation continue et si celle-ci joue le même rôle pour les deux sexes en termes de salaires.

\subsubsection{Des taux d'accès aux formations similaires...}

Dans les équations de formation, les variables de sexe ne sont pas statistiquement significatives, indiquant qu'à caractéristiques individuelles et professionnelles similaires, les hommes et les femmes ont des taux moyens identiques de participation aux formations continues. Nos résultats confirment ceux obtenus récemment par Turcotte et al. (2003) et Havet (2006) sur données canadiennes. Comme ces travaux, l'enquête Formation Continue 2000 a permis de retenir comme déterminants des décisions de formation des variables reflétant les stratégies d'organisation et de gestion de la main d'œuvre, souvent non prises en compte car non renseignées. L'accès différencié à la formation continue mis en évidence dans certaines études pourrait simplement refléter des différences hommes/femmes dans des caractéristiques non observées (ex : mises en place de nouveaux équipements, d'une nouvelle organisation, d'un aménagement du temps de travail) que de réels écarts dans le taux de formation, ceteris paribus.

En outre, lorsque l'on ne tient pas compte de la corrélation $\sigma_{12}$ entre les résidus des équations de formation, le coefficient associé au sexe devient significatif dans l'équation (1) laissant penser que les hommes suivent davantage de formation formelle que les femmes. Or, leur facilité d'accès proviendrait uniquement de leurs caractéristiques personnelles inobservables.

\footnotetext{
${ }^{7}$ Parmi les travaux corrigeant l'endogénéité et/ou l'hétérogénéité inobservée, la borne inférieure du rendement de la participation à la formation est donnée par Schone (2004) pour la Norvège et Gerfin (2004) pour l'Allemagne avec $1 \%$ à $2 \%$ alors que la borne supérieure est de l'ordre de $20 \%$ selon Groot (1995) pour les Pays-Bas, Regner (2002) pour la Suède ou Kuckulenz \& Zwick (2003) pour l'Allemagne. Voir aussi le tableau récapitulatif de Bassanini et al. (2007).
} 


\subsection{2 … mais des répercussions salariales différenciées}

Toutefois, les rendements salariaux des deux types de formation continue ne sont pas parfaitement identiques entre hommes et femmes. Certes, le coefficient $\hat{\alpha}_{2}$ n'est pas statistiquement significatif indiquant que les hommes ne valorisent pas mieux leur participation à une formation informelle que les femmes, toutes choses étant égales par ailleurs. Ainsi, les femmes auraient autant de chances que les hommes d'obtenir une formation informelle et celles qui ont l'opportunité d'en recevoir une peuvent s'attendre à une augmentation de salaire comparable à celle de leurs homologues masculins. Les pratiques des employeurs en termes d'accès et de valorisation de la formation informelle ne semblent donc pas pouvoir être remises en cause du point de vue de l'égalité hommes/femmes.

En revanche, les femmes jouiraient d'une meilleure rentabilité de leur participation à une formation formelle que les hommes. Plus précisément, le fait de suivre une formation formelle augmente en moyenne la rémunération des femmes de 7,4\%. Les hommes qui participent à ce type de formation voient leur revenu seulement augmenter de $3,3 \%$.

Ces résultats pour la France confortent partiellement des études antérieures pour d'autres pays. Kuckulenz \& Zwick (2003) sur données allemandes et Havet (2006) sur données canadiennes ont mis en évidence aucune différence entre sexes dans les rendements salariaux des formations financées par les employeurs. Néanmoins, Blundell et al. (1996) pour la GrandeBretagne et Pischke (2001) pour l'Allemagne avaient déjà montré que la valorisation salariale des formations continues était plus importante pour les femmes que les hommes, notamment pour les cours structurés menant à une qualification.

Comme les entreprises françaises semblent mieux récompenser les efforts de formation formelle des femmes que des hommes, ce type de formation pourrait être un moyen à privilégier pour réduire les inégalités salariales entre sexes. En effet, il ressort de notre estimation que les femmes ont en moyenne, à caractéristiques individuelles et professionnelles comparables, des rémunérations inférieures de $15 \%$ à celles des hommes. La formation formelle aurait pour impact de réduire cet écart d'environ $4 \%$.

\section{Conclusion}

Cet article propose une étude de l'impact de la participation à une formation en entreprise sur les salaires, en détaillant les différences de valorisation entre sexes. Les données françaises de l'enquête Formation continue 2000 permettent de distinguer les formations continues formelle et informelle, ce qui offre la possibilité d'examiner les liens entre elles, de comparer leurs influences respectives sur les salaires et de savoir s'il existe des différences entre hommes et femmes dans la nature et les rendements des formations suivies.

Pour ce faire, on a estimé un système d'équations simultanées, qui modélise conjointement la participation aux deux types de formations continues et les salaires et qui tient compte des 
effets corrélés d'hétérogénéité individuelle. L'avantage de cette modélisation est qu'elle permet d'identifier les caractéristiques individuelles favorables à l'accès aux formations et de savoir si les suppléments salariaux associés à ces formations sont réellement à attribuer à la participation à ces programmes ou plutôt à un phénomène de sélection. La distinction entre l'impact propre des formations et le phénomène de sélection endogène est un cadre d'investigation nécessaire pour préconiser des politiques de gestion des ressources humaines efficaces. En particulier, si le mécanisme de sélection prédomine, ce sont principalement des caractéristiques personnelles particulières des participants aux formations (diplôme, poste occupé, etc) qui expliquent les rendements de tels programmes. Une stratégie équitable pour les pouvoirs publics serait alors de promouvoir un système de formation continue incitant les entreprises à cibler leurs actions sur les groupes qui ont les plus grandes difficultés à y accéder.

Nos estimations réalisées sur données françaises pour la période 1999-2000 montrent que la formation en entreprise est un élément prépondérant dans les carrières salariales individuelles. Elles confirment que les décisions concernant les formations continues et les salaires se prennent souvent de manière concomitante, d'où la nécessité d'utiliser des techniques économétriques tenant compte de ces interdépendances. Une part importante de l'écart de salaire moyen observé entre ceux ayant suivi une formation professionnelle (formelle ou informelle) et ceux n'en ayant pas suivi est attribuable au fait que les travailleurs les plus souvent choisis pour participer aux programmes de formation sont ceux qui ont les compétences observées et inobservées les plus élevées. Néanmoins, une fois cette hétérogénéité prise en compte, il persiste une différence de salaire non négligeable en faveur des travailleurs ayant suivi une formation professionnelle toutes choses étant égales par ailleurs.

D'un point de vue pratique, il apparaît aussi indispensable de distinguer les différents types de formation en entreprise dans les estimations de rentabilité. Les formations formelle et informelle ont des impacts bien distincts en termes d'augmentations salariales et notamment pour les hommes et les femmes. Parmi les hommes, la formation informelle semble avoir une meilleure valorisation que la formation formelle, alors que le résultat est inverse parmi les femmes. Par ailleurs, il ressort qu'en France, le rendement d'une formation formelle est plus élevé pour les femmes que leurs homologues masculins et que le rendement d'une formation informelle est équivalent pour les deux sexes. En conséquence, il serait davantage recommandé de développer des cours de formation structurée que des formations informelles afin de limiter les disparités salariales entre les hommes et femmes. Comme le laissaient déjà entrevoir les études de Blundell et al. (1996) ou de Havet (2006), il semble que plus le contenu de la formation proposée est précis, et plus les compétences acquises et les progrès individuels peuvent être facilement évalués voire même se solder par une qualification répertoriée, plus l'accroissement de productivité a des chances d'être reconnu et récompensé en termes de salaires. Les employeurs se basent alors davantage sur des critères objectifs que sur des préjugés ou autres critères défavorables aux femmes.

A en croire nos résultats, on pourrait s'attendre à une réduction de l'écart de salaire observé entre hommes et femmes ceteris paribus dans l'avenir puisque le taux d'accès aux formations continues est identique pour les deux sexes mais leurs rendements supérieurs pour les femmes. 
En outre, on a montré que les travailleurs ayant une formation initiale la plus avancée sont les plus susceptibles de suivre une formation professionnelle. Or actuellement, on observe en France que les succès scolaires et universitaires des jeunes femmes sont supérieurs à ceux de leurs homologues masculins. Toutefois, la formation continue ne pourra être un moyen de réduire notablement les inégalités salariales entre sexes que si parallèlement les femmes ont accès aux mêmes types d'emploi que les hommes : la position de l'emploi occupé dans la hiérarchie (cadre, ouvrier, etc) est en effet déterminante dans l'accès aux formations continues. 


\section{Références}

Acemoglu, D. \& Pischke, J. (1999), 'Beyond becker : Training in imperfect labor market', Economic Journal 109, 112-142.

Arulampalam, W. \& Booth, A. (1998), 'Training and labour market flexibility : is there a trade-off?', British Journal of Industrial Relations 36(4), 521-536.

Barrett, A. \& O'Connell, P. (2001), 'Does training generally work? the return to in-company training', Industrial and Labor Relations Review 54(3), 647-663.

Bartel, A. (1995), 'Training, wage growth, and job performance : Evidence from a company database', Journal of Labor Economics 13(3), 401-425.

Bassanini, A., Booth, A., Brunello, G., Paola, M. D. \& Leuven, E. (2007), Workplace training in europe, in Brunello, Garibaldi \& Wasmer, eds, 'Education and Training in Europe', Oxford : Oxford University Press, chapter 8-13.

Becker, G. (1964), Human Capital. A Theoretical and Empirical Analysis with Special Reference to Education, New York and London : Columbia University Press.

Black, S. \& Lynch, L. (1998), 'Beyond the incidence of employer-provided training', Industrial and Labor Relations Review 52(1), 64-81.

Black, S. \& Lynch, L. (2001), 'How to compete : The impact of workplace practices and information technology on productivity', Review of Economics and Statistics 83(3), 434-445.

Blundell, R., Dearden, L. \& Meghir, C. (1996), The determinants and effects of work-related training in britain, Technical report, Institute for Fiscal Studies, London.

Blundell, R. \& Meghir, C. (1999), Work-related training and earnings, Technical report, Institute for Fiscal Studies.

Booth, A. \& Bryan, M. (2007), 'Who pays for general training? new evidence for British men and women', Research in Labor Economics 26, 85-123.

Cahuc, P. \& Zylberberg, A. (2006), La formation professionnelle des adultes : un système à la dérive, Technical report.

Chang, C. \& Wang, Y. (1996), 'Human capital investment under asymetric information : The pigovian conjecture revisited', Journal of Labor Economics 14(3), 505-519.

Corcoran, M. \& Duncan, G. (1979), 'Work history, labor force attachment, and earnings differences between the sexes', Journal of Human Resources 14(1), 3-20.

Coulombe, S., Tremblay, J.-F. \& Marchand, S. (2004), 'Literacy scores, human capital and growth across fourteen oecd countries', Cahier de recherche, Statistique Canada no. 89-552-MIE.

Delame, E. \& Kramarz, F. (1997), 'Entreprises et formation continue', Economie et Prévision 127(1), 63-82.

Fougère, D., Goux, D. \& Maurin, E. (2001), 'Formation continue et carrières salariales', Annales d'Économie et de Statistique 62, 49-69.

Gauron, A. (2000), Formation tout au long de la vie, in 'Rapport du Conseil d'Analyse économique', Vol. 22, La documentation française : Paris. 
Gerfin, M. (2004), 'Work-related training and wages : An empirical analysis for male workers in switzerland', IZA Discussion Papers 1078.

Goux, D. \& Maurin, E. (2000), 'Returns to firm-provided training : Evidence from french worker-firm matched data', Labour Economics 17, 1-19.

Gronau, R. (1988), 'Sex-related wage differentials and women's interrupted careers - the chicken or the egg?', Journal of Labor Economics 6(3), 277-301.

Groot, W. (1995), 'The wage effect of investments in enterprise-related training', Empirical Economics 20, 133-147.

Havet, N. (2006), 'La valorisation salariale et professionnelle de la formation en entreprise diffère-t-elle selon le sexe? l'exemple canadien', Economie et Prévision 175-176(4-5), 147-161.

Heckman, J. (2000), 'Policies to foster human capital', Research in Economics 54, 3-56.

Hill, E. (2001), 'Post-school-age training among women : Training methods and labor market outcomes at older ages', Economics of Education Review 20(2), 181-191.

Kuckulenz, A. \& Zwick, T. (2003), 'The impact of training on earnings - differences between participant groups and training forms', ZEW Discussion Papers 03-06.

Lengermann, P. (1999), 'How long do the benefits of training last? evidence of long terms effects accross current and previous employers', Research in Labor Economics 18, 439-461.

Leuven, E. (2005), 'The economics of private sector training : A survey of the literature', Journal of Economic Survey 19(1), 91-111.

Leuven, E. \& Oosterbeek, H. (2008), 'An alternative approach to estimate the wage returns to private-sector training', Journal of Applied Econometrics 23, 423-434.

Lillard, L. \& Tan, H. (1992), Private sector training : Who gets it and what are its effects?, in R. Ehrenberg, ed., 'Reserach in Labor Economics', Vol. 13, Grennwich, Conn. and London : JAI Press, pp. 1-62.

Lynch, L. (1992), 'Private-sector training and the earnings of young workers', American Economic Review 82(1), 299-312.

Maddala, G. (1983), Limited Dependent and Qualitative Variables in Econometrics, Cambridge University Press.

OCDE (1995), L'étude de l'OCDE sur l'emploi, Partie II : possibilités d'adaptation des marchés du travail, OCDE, Paris.

Perez, C. \& Thomas, G. (2005), 'Trajectoires d'emploi précaire et formation continue', Economie et Statistique 388-389, 107-127.

Pischke, J.-S. (2001), 'Continuous training in germany', Journal of Population Economics 14(3), 523-548.

Regner, H. (2002), 'The effects of on-the-job training on wages in sweden', International Journal of Manpower 23(4), 326-344.

Schone, P. (2004), 'Why is the return to training so high ?', Labour 18(3), 363-378.

Turcotte, J., Léonard, A. \& Montmarquette, C. (2003), 'Nouveaux résultats sur les déterminants de la formation dans les emplacements canadiens', La série sur le milieu de travail en évolution, Statistique Canada 71-584-MIE(5). 
Veum, J. (1996), 'Gender and race differences in company training', Industrial Relations 35(1), 32-44. 